DOI: https://doi.org/10.46296/yc.v4i6edesp.0029

\title{
APLICACIÓN DE REGLAS DE ASOCIACIÓN SOBRE MICROSERVICIOS EN LAS MICROEMPRESAS.
}

\section{APPLICATION OF ASSOCIATION RULES ON MICRO-SERVICES IN MICRO-ENTERPRISES.}

\author{
Cevallos-Macías John ${ }^{1 *}$; Solórzano-Cadena Rubén²; Palma-Menéndez Susana ${ }^{3}$; \\ Verduga-Urdánigo Fabricio ${ }^{4}$ \\ ${ }^{1}$ Universidad Laica "Eloy Alfaro" de Manabí. Manta, Ecuador. \\ ¿Universidad Laica "Eloy Alfaro" de Manabí. Manta, Ecuador. \\ "Universidad Laica "Eloy Alfaro" de Manabí. Manta, Ecuador. \\ "Universidad Laica "Eloy Alfaro" de Manabí. Manta, Ecuador.
}

*Correo: john.cevallos@uleam.edu.ec

\begin{abstract}
Resumen
Las microempresas son un grupo tecnológicamente desatendido debido a sus recursos limitados y carencia de conocimiento tecnológico que en muchos casos impide su crecimiento como negocio. En la presente investigación se diseña una estructura de minería de datos que basada en reglas de asociación ofrece sugerencias de compras mediante un dispositivo móvil implementando tácticas de up-selling y cross-selling. El diseño de la propuesta se basa en el proceso de generación de modelos de minería de datos y culmina con la validación e implementación de modelos de reglas de asociación como microservicios orquestados por un Web Service, esta arquitectura de servicios se encuentra desarrollada en Node.js, y se conecta con una base de datos PostgreSQL, mientras que la aplicación móvil se desarrolló en lonic Framework que le permite estar disponible para varias plataformas. La herramienta móvil presenta un aporte a los negocios informales, en los que un vendedor atiende directamente a los clientes y brinda alternativas de compra incrementando sus ventas en un porcentaje cercano al $25 \%$, además de otros beneficios menos tangibles como la mejora en la atención al cliente en los negocios que carecen de infraestructura tecnológica.
\end{abstract}

Palabras clave: reglas de decisión, sistemas de recomendación, microempresa, microservicios.

\begin{abstract}
The microenterprises are a technologically neglected group due to their limited resources and lack of technological knowledge that in many cases prevent their growth as a business. This research is based in a data mining structure with association rules that offers shopping suggestions using a mobile device and implementing up-selling and cross-selling tactics, given those points, the design of the proposal is based on the process of generation of data mining models and culminates with the validation and implementation of rules for the association of microservices or servers by a web service, this service architecture is developed in Node. js, and it connects with a PostgreSQL database, while the mobile application was developed on lonic framework, such that it is available for several platforms. Additionally, the mobile tool presents a contribution to informal businesses, where a seller serves customers directly and provides purchase alternatives increasing their sales by a percentage close to $25 \%$. Similarly, other less tangible benefits such as improving customer service in the businesses without technological infrastructure.
\end{abstract}

Keywords: Association rules, recommender system, engine, microservices.

Información del manuscrito:

Fecha de recepción: 21 de febrero de 2020

Fecha de aceptación: 20 de abril de 2020

Fecha de publicación: 05 de mayo de 2020 


\section{Introducción}

Existen una gran variedad de algoritmos orientados a relacionar hechos en común y entregar conocimiento a los sistemas de recomendación, aquellos algoritmos son utilizados de diversas formas y en algunos casos mejorados según la situación en particular. Los campos comerciales a los que se puede aplicar los sistemas de recomendación son cada vez más, lo que se podría presentar como una idea a reutilizar y difundir sin tener que entrar en detalles técnicos.

La heterogeneidad de los hechos y atributos que pueden afectar la toma de decisiones, representa que se realicen soluciones parciales y poco reutilizables; por lo que normalizar los parámetros de los algoritmos en una estructura jerárquica, y abstraer el funcionamiento de los algoritmos de decisión orientados a la recomendación de opciones en un servicio web, nos va a permitir constatar si los sistemas de recomendación pueden expandir su uso a múltiples empresas medianas y pequeñas, con productos y servicios de características diferenciables y líneas variadas; así apoyar el recurso humano dedicado a las ventas con una herramienta de alta usabilidad, e instalable sobre todo tipo de dispositivo que soporte la implementación de servicios web.

La solución propuesta es un servicio web de tipo REST que abstraiga y encapsule la lógica e implementación de los algoritmos de aprendizaje automático, necesarios para realizar recomendaciones de productos afines; este servicio web recibe como parámetros datos que respetan una jerarquía de atributos genéricos previamente definidos, además de la ubicación de los datos que serán contrastados con los algoritmos de asociación como Apriori, fp-growth, Eclat, etc. (School, 2017).

Para monitorear, analizar y validar los resultados, se implementará el web service como un módulo de soporte sobre 3 líneas distintas: ropa de bebe, accesorios maternos y productos de higiene. Para el análisis de la efectividad se implementa una solución móvil que utilizan los vendedores como apoyo a las tácticas de marketing crossselling y up-selling, midiendo los aciertos de las recomendaciones y la usabilidad de la aplicación al 
momento de solicitar una recomendación.

\subsection{Importancia del Problema}

Las aplicaciones y plataformas desarrolladas para la venta de productos y servicios, son muy utilizadas en pequeñas y medianas empresas a nivel latinoamericano, se ha convertido en un nicho de mercado, tanto para las empresas que emprenden en el desarrollo de software y necesitan capitalizar obteniendo ganancias a corto plazo, como para las grandes empresas desarrolladoras de software, que elaboran sofisticados sistemas de planificación de recursos empresariales (ERP) y/o aplicaciones de venta en línea sobre plataformas tanto web como móvil. Muy pocas de estas aplicaciones cuentan con módulos para recomendación de productos, y las que lo poseen, presentan un algoritmo orientado al marketing y la fidelización de clientes. Estos algoritmos no se encuentran estandarizados, ni existe una formalidad al momento de evaluar que atributos ni en que formato son enviados, así mismo sus resultados, los productos recomendados a su solicitante y el grado de certeza de las alternativas.

La arquitectura más utilizada para sistemas heterogéneos se orienta a servicios web, ya que utilizándolos se pueden servir datos a múltiples plataformas y tecnologías que los implementan, así también brindar estandarización a múltiples clientes y mejorar la productividad a nivel macro. Este trabajo integra algoritmos de aprendizaje automático en esta arquitectura y encapsula muchas decisiones colaterales al momento de recomendar un producto $o$ un servicio. Replicando la utilización de este servicio web, podemos garantizar que una gran cantidad de desarrolladores puedan contar con algoritmos de inteligencia en sus aplicaciones con características escalables y recursos centralizados.

Las tecnologías de la información influyen positivamente en la productividad, gestión de los procesos y sobre todo en la gestión de los clientes (Edgar Julián Gálvez Albarracín, 2014) en las micro, pequeñas y medianas empresas (MIPYMES), en dicho estudio desarrollado en Colombia se muestra mediante datos estadísticos 
la mejora significativa en la satisfacción del cliente y los procesos en general al incluir tecnologías de la información. Una conclusión relevante es que tanto la edad como el tamaño de la empresa al momento de beneficiarse de entornos web no son factores para considerar, indicando que estas aplicaciones pueden beneficiar de manera general a todo tipo de empresas por más pequeñas y nuevas que sean. La siguiente investigación (Mireya Flores Jaén, 2016) realizada a empresas en la ciudad de Quevedo detalla algunas características que frecuentemente son causas en la escasez de productos tecnológicos dirigidos a este medio. Entre los principales problemas de crecimiento tecnológico se encuentra la falta de financiamiento, la escasez de recurso humano con conocimiento informático, mucha competencia local y en muchas ocasiones la carencia de interés en este tipo de inversión. En este artículo se presenta adicionalmente un análisis sectorial relevante en el que sintetizando podemos deducir que la mayoría de las pymes presentan informalidad contable, capital limitado y gastos corrientes como arriendos que acrecientan el problema, carecen de una estructura organizacional, buscan la calidad como estrategia para mejorar sus negocios, pero no se incluye la tecnología para alcanzarla.

Otro de los inconvenientes encontrados en el comercio, cuando interactuamos como un mercado informal es la alta rotación tanto laboral como de productos (Ignacio Apella, 2008), provocando que los artículos como tal no tengan un historial constante (Chris Cornelis, 2007) y necesiten entrar en los sistemas de recomendación basados en el contenido, en este caso el contenido está apoyado en atributos, características y medidas de similitud. Una de las medidas tomadas por las microempresas para mantener la competitividad es pertenecer a una red de cooperación (Benito Hernández, 2009), estas redes o grupos de empresas se apoyan en diversos campos incluyendo el tecnológico, y se ha comprobado que este tipo de cooperación realmente puede incrementar el porcentaje de implementación de tecnologías de la información, siendo la mejorar arquitectura tecnológica la de 
servicios en un escenario de cooperación.

El $82 \%$ de las pequeñas y medianas empresas (PYMES) en el Ecuador tienen acceso a Internet (Mónica Orozco, 2015), pero no le sacan el provecho adecuado, implementando aplicaciones que incrementen la calidad de sus servicios, por lo que el hardware sería el costo de inversión al que debemos disminuir, encontrando una opción económica, pero con el desempeño adecuado, como es el uso de tabletas de bajo costo; existen tabletas consideradas económicas(Navarro, 2018), y mucho más accesible que otro tipo de hardware. Adquiriendo dispositivos móviles y utilizando el internet podemos implementar aplicaciones tecnológicas actualizadas, de bajo costo y de gran utilidad empresarial.

\subsection{Caso de estudio}

Manabí provincia de Ecuador, sufrió un fuerte impacto comercial con el terremoto del 16 de abril del 2017 (Universo, 2016), provocando un problema económico muy graveen la provincia y generando pérdidas de al menos $\$ 860$ millones al $49 \%$ del sector productivo. En Manta, el sector de Tarqui donde se asentaban
4200 comerciantes sufrió graves daños estructurales, impidiéndoles seguir utilizando el sector para sus ventas y sin poder contar con un crédito para reactivarse por falta de capital, creando una brecha muy difícil de superar para los microempresarios y emprendedores. Estos problemas fueron aplacados creando una nueva zona comercial denominada Nuevo Tarqui (Comercio, 2017), generando nuevas plazas de desarrollo tanto para el comercio minorista como al mayorista.

La arquitectura y sus servicios serán aplicados como una solución dirigida al sector comercial minorista y mayorista en desarrollo, teniendo en cuenta que en este caso de estudio los comerciantes están en proceso de resurgimiento y valorando la importante de este sector a la economía de la provincia (López, 2017), ya que según los datos de la INEC el $93 \%$ de las empresas que sostienen la economía son microempresas y un $36 \%$ de estas son comerciales. En la figura 1, tomado del artículo, podemos observar la distribución de empresas en la provincia, siendo Manta y Portoviejo pilares de la economía y de mayor relevancia para las cifras 
económicas locales, sector que fue el más afectado con la disminución del $29 \%$ de compras de sus clientes a nivel de Manabí.

Figura 1. Número de empresas por Cantón en Manabí.

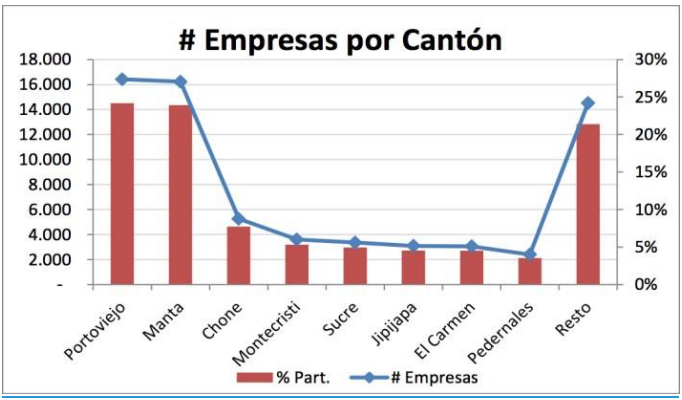

Fuente: INEC.

La herramienta creada toma a este sector comercial afectado por un terremoto como caso de estudio, comercio ahora ubicado en el parque comercial Nuevo Tarqui, este escenario careció de créditos e inyecciones de capital para retomar sus actividades, por lo que la tecnología a pesar de ser necesaria para reactivarse y brindar una nueva cara a los clientes no es de fácil acceso; el trabajo desarrollado brinda una aplicación que sistematiza las ventas, y recomendaciones apoyándose en servicios web alojados en el internet. La utilización de esta herramienta en este escenario demuestra que la implementación de microservicios, como un motor de recomendaciones, aplicando algoritmos personalizados al entorno es factible $y$ recomendable al sector comercial de las micro, pequeñas y medianas empresas.

\section{Metodología.}

De forma general los componentes, el desarrollo y las herramientas utilizadas para generar un servicio web que implemente los algoritmos de aprendizaje y sirva a la aplicación cliente las recomendaciones sobre que producto ofertar están plasmados en las siguientes etapas: elección de algoritmos a implementar, personalización de algoritmos, pruebas de ejecución de los servicios y sus algoritmos, implementación de servicios web, herramienta de aplicación y evaluación de recomendaciones y metodología utilizada en la evaluación de la herramienta.

\subsection{Elección de algoritmos}

Para la elección de los algoritmos se ha revisado la librería propuesta en la investigación (Philippe FournierViger A. G.-W., 2014), la cual presenta un gran número de soluciones implementadas en Java como software libre y enfocándose en la clasificación Frequent Itemset 
Mining que presta la solución utilizada en software de recomendación de productos. Esta librería se encuentra actualizada (Philippe Fournier-Viger J. C.-W., 2016) y cuenta con una interfaz gráfica, así como conjunto de datos para pruebas en formato texto plano. Los algoritmos de mayor utilización son fp-growth y apriori, con modificaciones en sus implementaciones basado en el entorno y el problema al que va dirigido. Por ejemplo (Youcef Djenouri, 2017) el algoritmo SS-FIM que tiene una gran similitud con Apriori puede obtener mejores resultados en conjuntos de datos grandes/ muy grandes disminuyendo el número de pasadas por las transacciones a una sola.

Aunque existen trabajos en los que el filtrado colaborativo por sí solo no representan una herramienta definitiva para brindar recomendaciones útiles (Chris Cornelis, 2007), debido a la existencia de ítems sin regularidad, que provocan carencia total de historial y seguimiento de preferencias. Presentando soluciones que no pueden ser aisladas, más bien son complementarias como la utilización del contexto. La elección de candidatos también puede ser modificada como en el caso de (Jie Bao, 2012) donde se plantea un algoritmo que tiene como dato de entrada la región espacial en adición a el usuario con sus selecciones previas.

El formato y la estructura como se trabaja la información es también relevante como en el trabajo de (CHANG TAN, 2014) al utilizar el par de atributo valor para definir las características de los elementos y proponiendo redes bayesianas para las similitudes entre estos; también la estructura de árbol es utilizada en conjuntos de datos más difusos (Dianshuang Wu G. Z., 2013) siendo los métodos de similitud entre sus nodos, los más estudiados y relevantes al momento de desarrollar un algoritmo.

Tomando en cuenta que los algoritmos más utilizada en los sistemas de recomendación siguen siendo los de filtrado colaborativo y que la variación en la implementación radica en el contexto y sus problemas subyacentes, se llegó a la conclusión que los algoritmos a implementar en el motor desarrollado serán los mismos que 
se han estudiado por su nivel de relevancia, pero con modificaciones enfocadas al momento de comparar los ítems. Además, se considera el formato que en este caso sería JSON, con objetos y sus atributos para realizar comparaciones utilizando funciones del lenguaje JavaScript.

\subsection{Pruebas de ejecución de los servicios}

Para una mayor explicación de cómo funcionan los microservicios, se detallan pruebas realizadas sobre los servicios y como estos responden dependiendo de los parámetros suministrados. Las llamadas al servicio principal son realizadas sobre el método POST, utilizando el método recomendar. Estos llamados funcionan como una sobrecarga de métodos, al recibir parámetros distintos en el cuerpo de las opciones que deben ir luego de la lista de ítems como parámetro obligatoriamente ubicado en el primer lugar. A continuación, la Tabla 1, muestra valores de ejemplo para los parámetros y la explicación de lo que representan para el servicio principal.
Tabla 1. Llamadas a servicio principal

\begin{tabular}{|c|c|c|}
\hline $\begin{array}{l}\text { Identif } \\
\text { icador }\end{array}$ & Parámetros & Explicación \\
\hline 15 & $\begin{array}{l}\text { \{listaltem:[\{iditem:133\}, } \\
\text { \{idltem:255\}, } \\
\text { \{idltem:900\}], } \\
\text { listaGrupoFiltro: } \\
\text { [\{idGrupo:2\}, } \\
\text { \{idGrupo:3\}]\} }\end{array}$ & $\begin{array}{l}\text { Se envía la lista de } \\
\text { ítems de la canasta } \\
\text { de compras del } \\
\text { cliente hasta el } \\
\text { momento, también } \\
\text { la lista de grupos } \\
\text { que el vendedor } \\
\text { seleccione con la } \\
\text { interacción con el } \\
\text { cliente. }\end{array}$ \\
\hline 17 & $\begin{array}{l}\text { \{listaltem:[\{idltem:133\}, } \\
\text { \{idltem:900\}], } \\
\text { listaCaracteristica: } \\
\text { [\{idGrupoltem:2\}, } \\
\text { \{idGrupoltem:4\}]\} }\end{array}$ & $\begin{array}{l}\text { La lista de ítems, y } \\
\text { adicionalmente una } \\
\text { lista de } \\
\text { características } \\
\text { relevantes. }\end{array}$ \\
\hline 18 & $\begin{array}{l}\{\text { listaltem:[\{idltem:255\}], } \\
\text { listaCaracteristicaMapa: } \\
\text { [\{ idGrupoltemMapa:3 \}, } \\
\{\text { idGrupoltemMapa:4\}]\} }\end{array}$ & $\begin{array}{l}\text { Lista de ítems, y } \\
\text { características de } \\
\text { mapeo relevante. }\end{array}$ \\
\hline
\end{tabular}

Fuente: Autores.

En el primer caso con el identificador 15 asignado, el servicio principal según sus condiciones al validar los atributos del segundo parámetro direcciona los parámetros al primer microservicio. El funcionamiento del servicio se detalla en Tabla 2, donde luego de ejecutar el algoritmo devuelve al servicio principal los ítems sugeridos según los datos frecuentes con soporte mayor preconfigurado como se muestra en la Tabla 3. 
Tabla 2. Llamada al primer microservicio.

\begin{tabular}{|c|c|c|c|}
\hline Método & Parámetro & Respuesta & Explicación \\
\hline $\begin{array}{l}\text { POST - } \\
\text { Llamar }\end{array}$ & $\begin{array}{l}\text { \{listaltem: } \\
\text { [\{iditem:133\}, } \\
\text { \{idltem:255\}, } \\
\text { \{idltem:900\}], } \\
\text { listaGrupoFiltro: } \\
\text { [\{idGrupo:2\}, } \\
\text { \{idGrupo:3\}]\} }\end{array}$ & $\begin{array}{l}\text { [\{id:200, } \\
\text { descripción: } \\
\text { 'Cuna BBs } \\
\text { Rojo- } \\
\text { Violeta'\}] }\end{array}$ & $\begin{array}{l}\text { Se envía los } \\
\text { identificadores } \\
\text { de los ítems } \\
\text { seleccionados } \\
\text { por el cliente y } \\
\text { grupos que en } \\
\text { este caso es } \\
\text { una madre } \\
\text { embarazada y } \\
\text { primeriza. El } \\
\text { método } \\
\text { retorna un } \\
\text { arreglo de } \\
\text { ítems que } \\
\text { cumplen las } \\
\text { reglas de } \\
\text { asociación. }\end{array}$ \\
\hline $\begin{array}{l}\text { GET - } \\
\text { Prueba }\end{array}$ & $\begin{array}{l}\text { No presenta } \\
\text { parámetros de } \\
\text { entrada }\end{array}$ & $\begin{array}{l}\text { (Estado: } \\
\text { Verdadero) }\end{array}$ & \\
\hline
\end{tabular}

Fuente: Autores.

Tabla 3. Conjunto de datos frecuente.

\begin{tabular}{|l|l|}
\hline Conjunto de datos & Soporte \\
\hline$\{133,255,900,126\}$ & 7 \\
$\{133,255,900,200\}$ & 2 \\
\hline
\end{tabular}

Fuente: Autores.

El identificador 17 detalla un ejemplo con diferentes parámetros al anterior, en el caso mostrado en la Tabla 4 se envía como parámetro la lista de características que se consideran relevantes para la comparación entre ítems, devolviendo un arreglo de ítems que cumplen la frecuencia de nodos a recomendar.
Tabla 4. Llamada al segundo microservicio.

\begin{tabular}{|c|c|c|c|}
\hline Método & Parámetro & Respuest & Explicación \\
\hline $\begin{array}{l}\text { POST - } \\
\text { Llamar }\end{array}$ & $\begin{array}{l}\text { \{listaltem: } \\
\text { [\{iditem:133\}, } \\
\text { \{idltem:900\}], } \\
\text { listaCaracterist } \\
\text { ica: } \\
\text { [\{idGrupoltem: } \\
2\}, \\
\{\text { idGrupoltem: } \\
4\}]\}\end{array}$ & $\begin{array}{l}\text { [\{id:335, } \\
\text { descripció } \\
\text { n: } \\
\text { 'Andador } \\
\text { de bebe } \\
\text { azul } \\
\text { Mickey - } \\
\text { seguro } \\
\text { plástico'\}] }\end{array}$ & $\begin{array}{l}\text { Se envía los } \\
\text { identificadores } \\
\text { de los ítems } \\
\text { seleccionados } \\
\text { por el cliente y } \\
\text { las } \\
\text { características } \\
\text { más relevantes } \\
\text { seleccionadas. } \\
\text { El método } \\
\text { retorna un } \\
\text { arreglo de } \\
\text { ítems que } \\
\text { cumplen con } \\
\text { las reglas de } \\
\text { frecuencia en } \\
\text { el árbol. }\end{array}$ \\
\hline $\begin{array}{l}\text { GET - } \\
\text { Prueba }\end{array}$ & $\begin{array}{l}\text { No presenta } \\
\text { parámetros de } \\
\text { entrada }\end{array}$ & $\begin{array}{l}\text { \{Estado: } \\
\text { Verdadero }\end{array}$ & \\
\hline
\end{tabular}

Fuente: Autores.

Por último, el caso del identificador 18 explicado en la Tabla 5 se diferencia por el nombre de los atributos, ya que también requiere una lista de características relevantes con la diferencia de que estos atributos serán mapeados utilizando un esquema equivalente al de la Tabla 6.

Tabla 5. Llamada al tercer microservicio.

\begin{tabular}{llll}
\hline Método & Parámetro & Respuesta & Explicación \\
\hline POST - & \{listaltem:[\{idl & [\{id:15, & Se envía los \\
Llamar & tem:255\}], & descripción & identificadores \\
& listaCaracteri & $:$ & de los ítems \\
sticaMapa: & 'Juguete & seleccionados \\
[\{ & Muñeca & por el cliente y \\
idGrupoltem & Maxi Perla'\}] & las \\
Mapa:3 $\},$ & & características \\
\{idGrupolte & & que deben ser \\
mMapa:4\}]\} & & mapeadas \\
& & para encontrar \\
& & el \\
& & Nivel de \\
& similitud. El
\end{tabular}




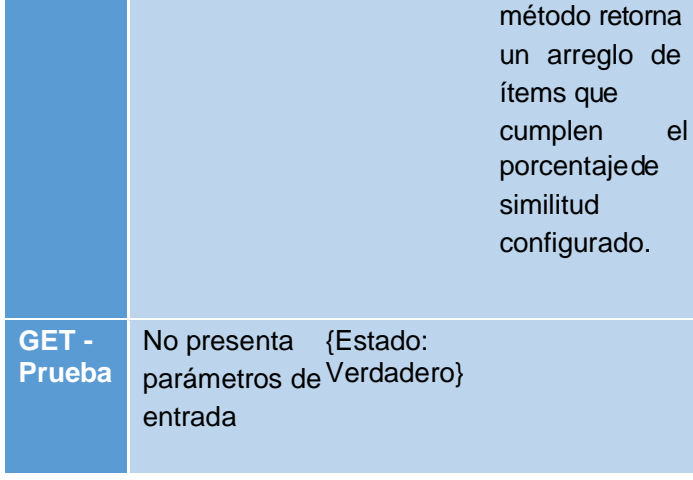

Fuente: Autores.

Tabla 6. Mapa de similitud en atributos.

\begin{tabular}{|lll|}
\hline Atributo A & Atributo B & $\begin{array}{l}\text { Porcentaje de } \\
\text { similitud }\end{array}$ \\
\hline Rosado & Rosa & 100 \\
\hline Rojo & Marrón & 90 \\
\hline Violeta & Azul & 60 \\
\hline Enconchado & Bordado & 70
\end{tabular}

Fuente: Autores.

\subsection{Implementación de servicios web}

La herramienta creada cuenta con un servicio web principal que recibe los artículos seleccionados por el cliente, e indicaciones por parte del vendedor que serán útiles según el algoritmo, como el tipo de cliente y su regularidad, estas indicaciones ayudan a tomar una decisión sobre que algoritmo se necesita y que microservicio utilizar. A continuación, en la Figura 2, podemos observar como el servicio de recomendación utiliza microservicios como bibliotecas para resolver sus requerimientos.
Figura 2. Arquitectura de microservicios.

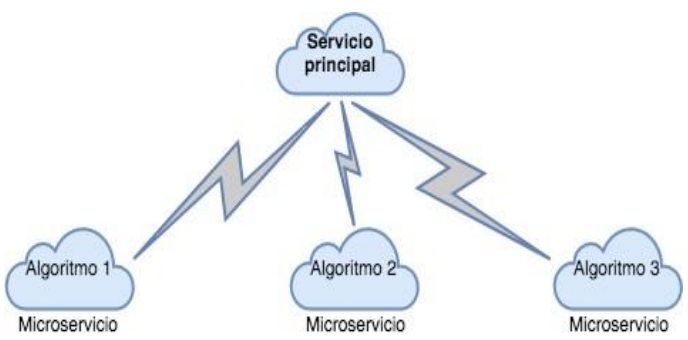

Fuente: Autores

La arquitectura es altamente escalable y tiene encomendadas 2 tareas principales: Escoger el algoritmo según parámetros y ejecutarlo, teniendo como entradas los ítems escogidos y los parámetros personalizados para la sugerencia. Estos parámetros o indicaciones son brindados por los vendedores en base a la observación, como en el caso de las características del cliente, o sistematizadas como la regularidad y fidelidad de un cliente. Como podemos ver en la Figura 3, otra de las salidas obtenidas es una traza en base al algoritmo ejecutado y el proceso llevado a cabo.

Figura 3. Tareas del servicio.

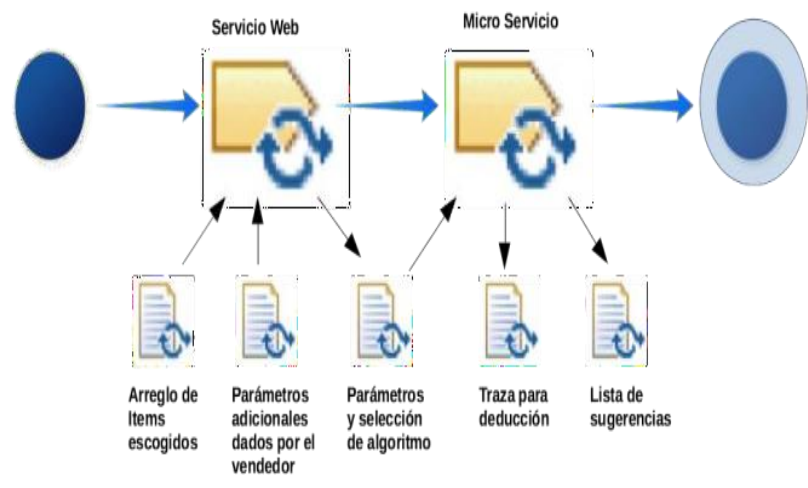

Fuente: Autores. 
A continuación, se detalla la definición de los microservicios, tanto el principal que funciona como Gateway como el de los algoritmos enmascarados como microservicios. El servicio REST principal que se muestra en la Tabla 7 tiene 2 métodos: el método recomendar que redirecciona a los otros servicios según los parámetros suministrados y el método Mostrar Traza que se encarga de mostrar información técnica de la recomendación previamente realizada.

Tabla 7. Servicio principal.

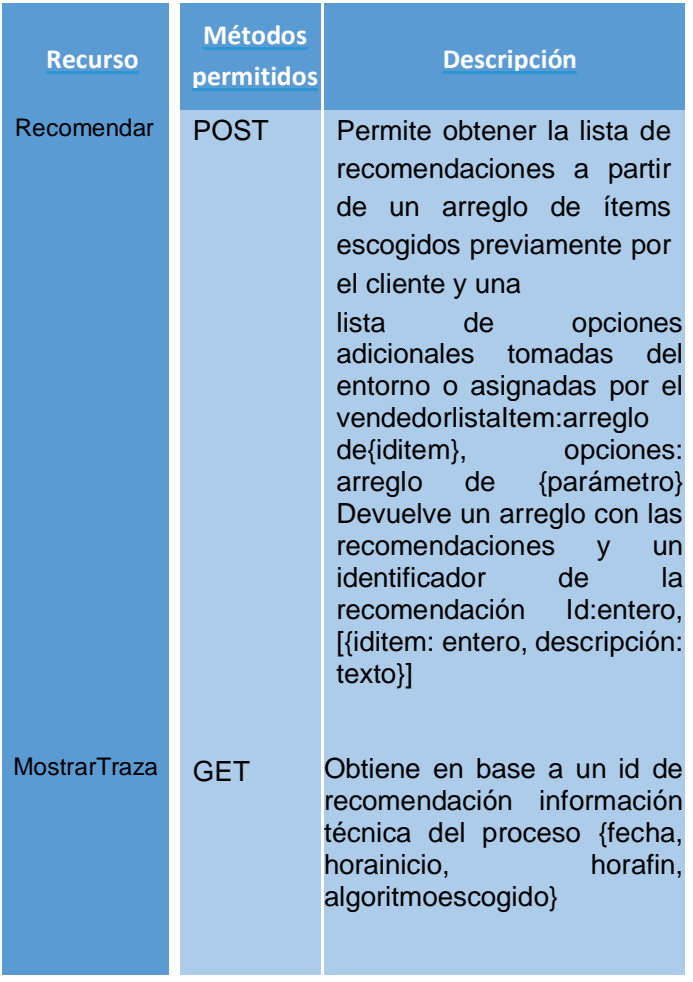

Fuente: Autores.

El primero algoritmo se encuentra incluido en la llamada al microservicio descrito en Tabla 8, para aquello es necesario que el vendedor incluya como parámetros algunas características como grupos de los clientes. También existe otro método que devuelve el estado del servicio, informando si este se encuentra disponible para su utilización.

Tabla 8. Servicio para algoritmo 1.

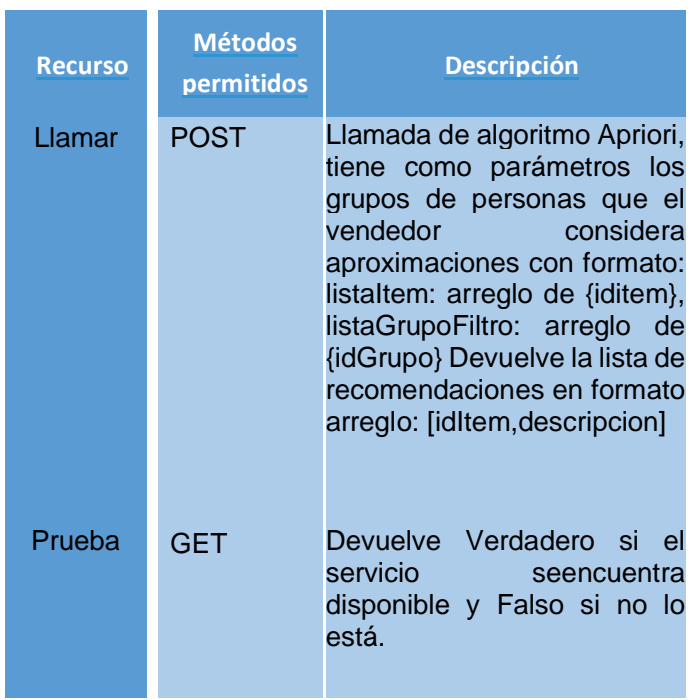

Fuente: Autores.

El segundo algoritmo está inmerso en la llamada al servicio descrito en la Tabla 9, presenta un método que devuelve las recomendaciones según los ítems previamente escogidos por el cliente y las características relevantes para la comparación utilizando el algoritmo. Presenta adicionalmente un método de prueba que se encarga de informar si este algoritmo se encuentra funcional. 
Tabla 9. Servicio para algoritmo 2.

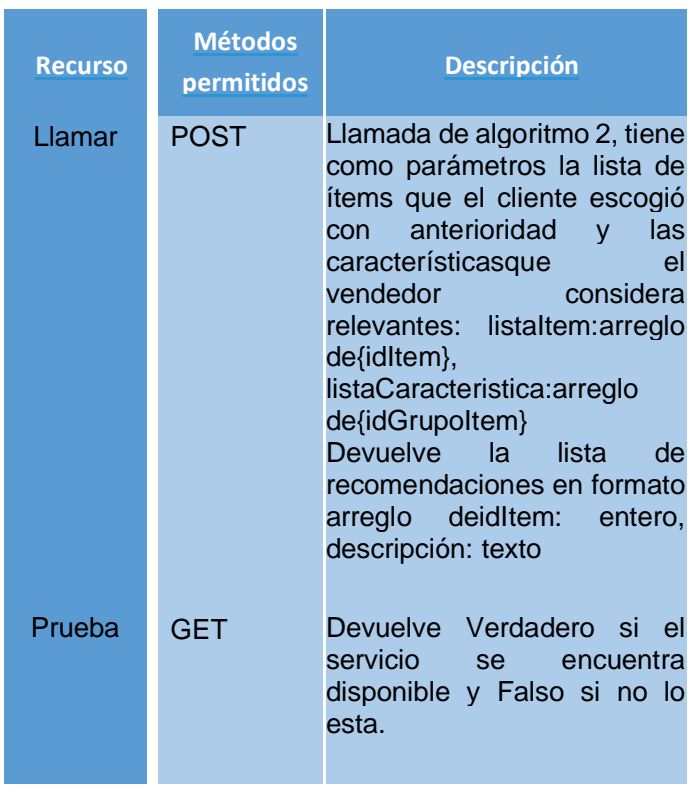

Fuente: Autores.

El servicio para el algoritmo 3 se encuentra descrito en la Tabla 10, con una estructura muy parecida al servicio anterior, ya que la diferencia radica en los pasos realizados por el algoritmo, más que en sus entradas y salidas. También presenta el método que prueba si el algoritmo se encuentra disponible y funcionando correctamente.

Tabla 10. Servicio para algoritmo 3.

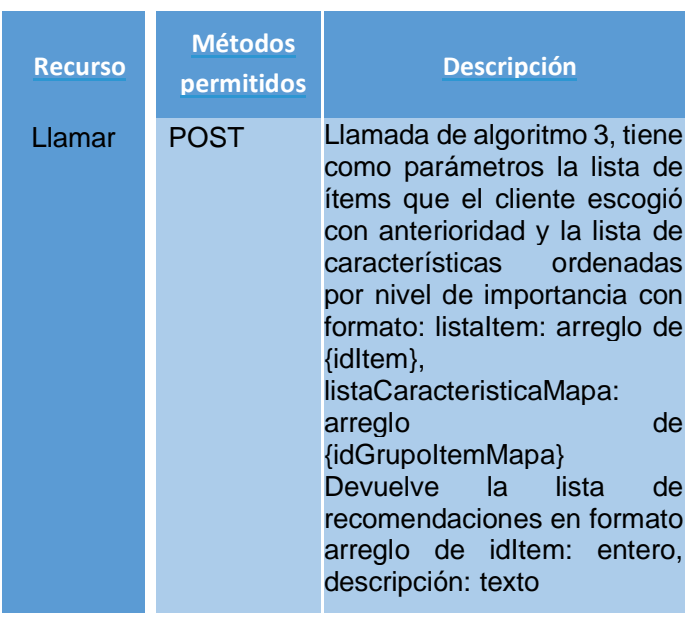

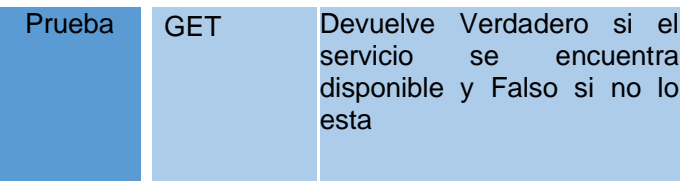

Fuente: Autores.

\subsection{Herramienta de evaluación de recomendaciones.}

Actualmente, la aplicación que se utiliza para evaluar las recomendaciones se encuentra desarrollada para ser ejecutado en el sistema operativo Android. No obstante, ha sido desarrollada de tal forma que puede ser portada al sistema operativo Apple iOS, así como a cualquier navegador web. Esta desarrollada utilizando Ionic (Dryfty, 2017), que integra algunas tecnologías como Angular, Typescripty las tecnologías por defecto para frontend.

Como característica de seguridad utiliza OAuth, implementada como se indica en la figura 4, en una interfaz de acceso a usuario, podemos notar como se abstrae la complejidad, ingresando únicamente usuario y clave.

Figura 4. Autenticación de usuario.

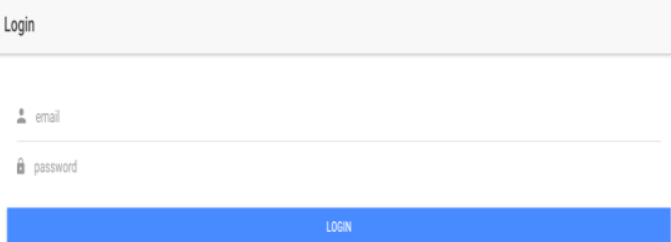

Fuente: Autores. 
La interfaz está diseñada para dispositivos móviles, sobre todo tabletas que serán las herramientas utilizadas por los vendedores. A continuación, en la figura 5 se muestra la interfaz principal de la aplicación, la cual permite escoger el vendedor que va a realizar la venta y la funcionabilidad para realizar un pedido de cliente.

Figura 5. Interfaz de pedidos.

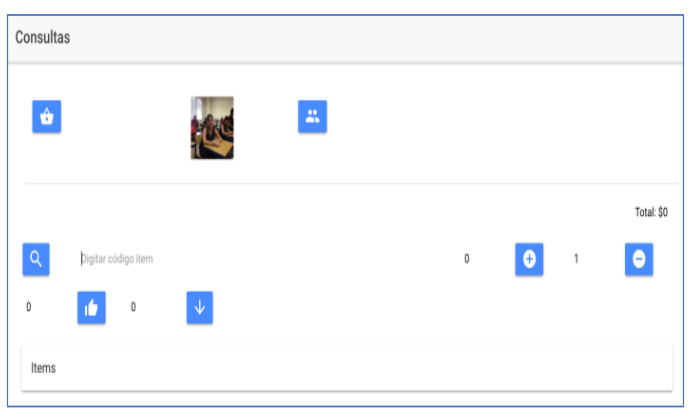

Fuente: Autores.

En la interfaz que se muestra en la figura 6 , podemos apreciar como al ingresar el código de un ítem para la venta, se muestran en un proceso asincrónico las recomendaciones según los factores de cada algoritmo o según el tipo de cliente escogido.

Figura 6. Consulta de recomendaciones.

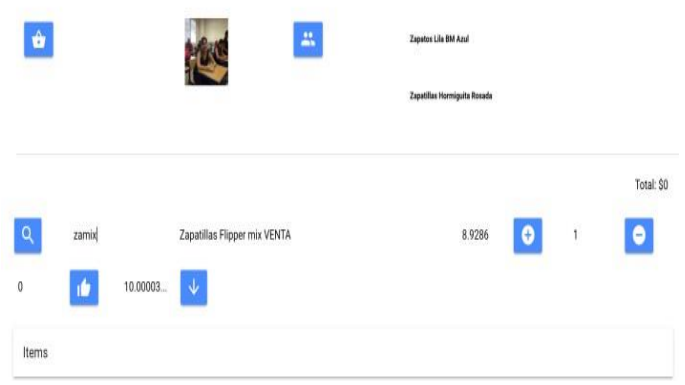

Fuente: Autores.
Para

poder

realizar

recomendaciones en negocios en los que la mayor cantidad de clientes tienen una frecuencia de ventas muy reducida, y otra parte de los clientes nunca han realizado un pedido, se categorizan los clientes según características visuales según se muestran en la figura 7 , que pueden ser captadas por los vendedores al momento de empezar la venta.

Figura 7. Categorización de clientes.

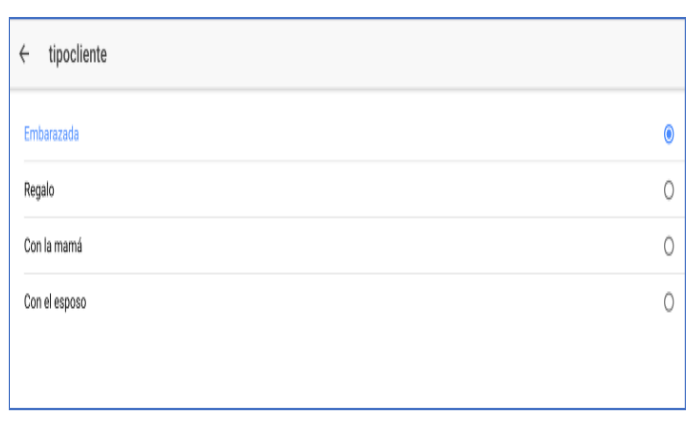

Fuente: Autores.

Al desear cambiar el vendedor actual se visualiza según la figura 8 , una interfaz que muestra las fotos de los vendedores acompañados de un gráfico estadístico con las comisiones generadas a partir de las recomendaciones. Esta interfaz fue creada para estimular el uso de las recomendaciones al personal de ventas, y la competencia entre ellos. 
Figura 8. Escoger vendedor.

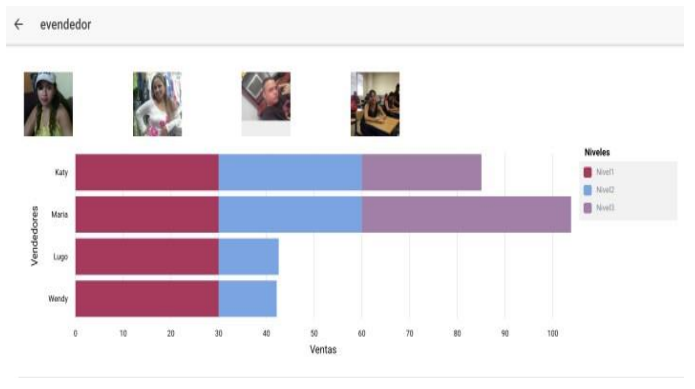

Fuente: Autores.

\section{Resultados y discusión.}

Se midió la usabilidad y la efectividad de los servicios web como herramienta de los sistemas de recomendación, utilizando metodologías y estándares definidos a continuación.

\subsection{Usabilidad}

Para medir la usabilidad, se ha tomado como referencia la ISO 25010 (25010, 2017), con su modelo para medir la calidad de un producto de software; tomando como referencia la característica de usabilidad y sus sub-características. El producto evaluado es el servicio web, y la aplicación móvil es el medio. Por aquello que se han considerado las características más orientadas al recurso obtenido, por parte del motor de recomendación y no a las interfaces. Por esto no son objeto de consideración características como: Accesibilidad y Estéticas de la interfaz.
Las características que han sido consideradas para la evaluación son: Inteligibilidad (Tabla 11), Operabilidad (Tabla 12) y Protección frente a errores de usuario (Tabla 13).

Tabla 11. Inteligibilidad.

\begin{tabular}{cl|l} 
Característica & \multicolumn{1}{c|}{ Atríbuto } & \multicolumn{1}{c}{ Significado } \\
\hline & $\begin{array}{l}\text { Actualización de ¿Sus } \\
\text { Componentes } \\
\text { no provoca } \\
\text { caídas en todo } \\
\text { carga de } \\
\text { trabajo }\end{array}$ & $\begin{array}{l}\text { el sistemalizables a } \\
\text { independiente? }\end{array}$ \\
& $\begin{array}{l}\text { Mantenibilidad } \\
\text { de software }\end{array}$ & $\begin{array}{l}\text { ¿Su software se } \\
\text { mantiene con } \\
\text { uso de internet? }\end{array}$ \\
& &
\end{tabular}

Fuente: Autores.

Tabla 12. Operabilidad.

\begin{tabular}{|c|ll}
\hline $\begin{array}{c}\text { Característica } \\
\text { Tiempo de }\end{array}$ & $\begin{array}{l}\text { Atributo } \\
\text { Tiempo de } \\
\text { Respuesta } \\
\text { al usuario }\end{array}$ & $\begin{array}{l}\text { ¿Es el tiempo de } \\
\text { respuesta óptimo } \\
\text { para satisfacer la } \\
\text { necesidad del } \\
\text { usuario? }\end{array}$ \\
\hline $\begin{aligned} \text { Posibilidades } \\
\text { de }\end{aligned}$ & $\begin{array}{l}\text { Nivel de } \\
\text { parametriza } \\
\text { ción }\end{array}$ & $\begin{array}{l}\text { ¿Nivel de } \\
\text { parametrización de } \\
\text { opciones? }\end{array}$ \\
\hline
\end{tabular}

Fuente: Autores.

Tabla 13. Protección a errores de usuario.

\begin{tabular}{|c|l|l|}
\hline $\begin{array}{c}\text { Característica } \\
\text { Recuperación } \\
\text { de errores }\end{array}$ & $\begin{array}{l}\text { Atributo } \\
\text { Proceso de } \\
\text { Recuperación } \\
\text { erros y fallas }\end{array}$ & $\begin{array}{l}\text { ¿Presenta } \\
\text { procesos de } \\
\text { recuperación al } \\
\text { suceder errores o } \\
\text { fallas? }\end{array}$ \\
\hline $\begin{array}{c}\text { Interfaz de } \\
\text { error }\end{array}$ & $\begin{array}{l}\text { Mensajes de } \\
\text { error } \\
\text { amigables a } \\
\text { nivel de } \\
\text { aplicación }\end{array}$ & $\begin{array}{l}\text { ¿Muestra errores } \\
\text { explícitos y } \\
\text { amigables en el } \\
\text { caso de existir? }\end{array}$ \\
\hline
\end{tabular}

Fuente: Autores. 
Las métricas han sido consideradas en base al significado de las características escogidas en el modelo, formando un cuestionario con respuestas cerradas tipo Si o No. A continuación, en la Tabla 14, se detallan las preguntas de la encuesta realizada, en base a las características y sub-características definidas según la ISO 25010:

Tabla 14. Preguntas de la encuesta.

\begin{tabular}{|c|c|}
\hline & Cuerpo de la encuesta \\
\hline Pregunta 1 & $\begin{array}{l}\text { ¿Cuándo se realizan cambios en los } \\
\text { algoritmos o actualizaciones al sistema } \\
\text { se presentan problemas de uso en } \\
\text { la aplicación móvil? }\end{array}$ \\
\hline Pregunta 2 & $\begin{array}{l}\text { ¿Las actualizaciones o mejores } \\
\text { requieren intervención humana sobre el } \\
\text { dispositivo móvil? }\end{array}$ \\
\hline Pregunta 3 & $\begin{array}{l}\text { ¿El tiempo de respuesta en que se } \\
\text { presentan las recomendaciones no } \\
\text { afecta la atención al cliente? }\end{array}$ \\
\hline Pregunta 4 & $\begin{array}{l}\text { ¿Es posible enviar parámetros desde la } \\
\text { aplicación que ayuden a que se realicen } \\
\text { mejores recomendaciones? }\end{array}$ \\
\hline Pregunta 5 & $\begin{array}{l}\text { ¿Cuándo se presentan errores en la } \\
\text { aplicación se suspende en tiempos } \\
\text { prolongados el uso de la aplicación? }\end{array}$ \\
\hline Pregunta 6 & $\begin{array}{l}\text { ¿Los mensajes obtenidos desde la } \\
\text { aplicación cuando se desea realizar } \\
\text { alguna recomendación es comprensible? }\end{array}$ \\
\hline
\end{tabular}

Fuente: Autores.

La encuesta diseñada fue realizada a los usuarios mostrados en la Tabla 15, que muestra el número de usuarios por cargo y la influencia que tiene la aplicación en su trabajo:
Tabla 15. Usuarios encuestados.

\begin{tabular}{|l|c|l|}
\hline \multicolumn{1}{|c|}{$\begin{array}{c}\text { Cargo del } \\
\text { usuario }\end{array}$} & $\begin{array}{c}\text { Número de } \\
\text { usuarios }\end{array}$ & $\begin{array}{c}\text { Influencia de } \\
\text { la aplicación }\end{array}$ \\
\hline Vendedor & 5 & Alto \\
\hline Técnico & 1 & Bajo \\
\hline $\begin{array}{l}\text { Administrador } \\
\text { Negocio }\end{array}$ & 2 & Alto \\
\hline
\end{tabular}

Fuente: Autores.

Los resultados generados para los usuarios son satisfactorios, en especial a los vendedores; a continuación, se detallan los resultados en la Tabla 16, con respuestas tabuladas por usuario.

Tabla 16. Respuestas tabuladas por usuarios.

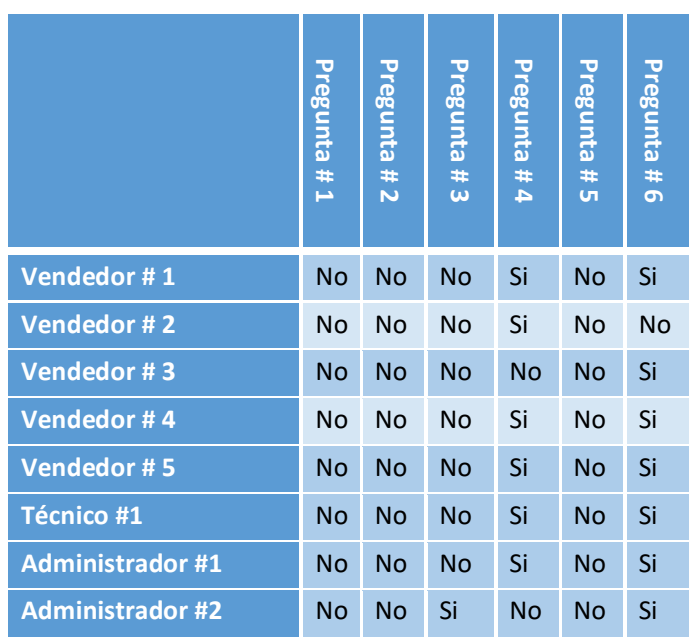

Fuente: Autores.

\subsection{Efectividad}

Para medir la efectividad se agregó una opción en la aplicación móvil, para que sea el vendedor en el momento de obtener las recomendaciones desde el motor, quien seleccione alguna de ellas, si le fue de interés al usuario alguna de 
las recomendaciones o producto afín queda a potestad del vendedor con un clic reconocerla como efectiva. Debemos tener en cuenta que el inventario es muy variable y los productos carecen de un historial extenso, por lo que es más valioso tener en cuenta las características de los ítems recomendados, que los productos en sí.

La medición fue realizada por fecha, según el número de clics realizados sobre los ítems recomendados por el servicio en contraste al número de ventas de esa fecha en particular, esto debido a que existen fechas de ventas muy superiores a otras. En la Tabla 17, que se muestra a continuación, se detallan los resultados de la medición sobre las ventas, en el lapso de un mes. Posteriormente procederemos a evaluar dichos resultados.

Tabla 17. Resultado de recomendaciones efectivas.

\begin{tabular}{|c|c|c|}
\hline Fecha & $\begin{array}{l}\text { Número de } \\
\text { ventas }\end{array}$ & $\begin{array}{l}\text { Número de } \\
\text { recomendaciones } \\
\text { efectivas }\end{array}$ \\
\hline $\begin{array}{l}\text { 01-diciembre- } \\
2017\end{array}$ & 33 & 6 \\
\hline $\begin{array}{l}\text { 02- diciembre- } \\
2017\end{array}$ & 64 & 7 \\
\hline $\begin{array}{l}\text { 03- diciembre- } \\
2017\end{array}$ & 32 & 4 \\
\hline $\begin{array}{l}\text { 04- diciembre- } \\
2017\end{array}$ & 21 & 3 \\
\hline $\begin{array}{l}\text { 05- diciembre- } \\
2017\end{array}$ & 23 & 5 \\
\hline $\begin{array}{l}06-\text { diciembre- } \\
2017\end{array}$ & 21 & 4 \\
\hline $\begin{array}{l}07-\text { diciembre- } \\
2017\end{array}$ & 31 & 5 \\
\hline
\end{tabular}

\begin{tabular}{|c|c|c|}
\hline $\begin{array}{l}08 \text { - diciembre- } \\
2017\end{array}$ & 39 & 9 \\
\hline $\begin{array}{l}\text { 09- diciembre- } \\
2017\end{array}$ & 99 & 25 \\
\hline $\begin{array}{l}\text { 10- diciembre- } \\
2017\end{array}$ & 36 & 6 \\
\hline $\begin{array}{l}\text { 11- diciembre- } \\
2017\end{array}$ & 42 & 8 \\
\hline $\begin{array}{l}\text { 12- diciembre- } \\
2017\end{array}$ & 32 & 7 \\
\hline $\begin{array}{l}\text { 13- diciembre- } \\
2017\end{array}$ & 48 & 8 \\
\hline $\begin{array}{l}\text { 14- diciembre- } \\
2017\end{array}$ & 29 & 6 \\
\hline $\begin{array}{l}\text { 15- diciembre- } \\
2017\end{array}$ & 54 & 11 \\
\hline $\begin{array}{l}\text { 16- diciembre- } \\
2017\end{array}$ & 147 & 45 \\
\hline $\begin{array}{l}\text { 17- diciembre- } \\
2017\end{array}$ & 97 & 23 \\
\hline $\begin{array}{l}\text { 18- diciembre- } \\
2017\end{array}$ & 72 & 22 \\
\hline $\begin{array}{l}\text { 19- diciembre- } \\
2017\end{array}$ & 71 & 20 \\
\hline $\begin{array}{l}20 \text { - diciembre- } \\
2017\end{array}$ & 96 & 31 \\
\hline $\begin{array}{l}\text { 21- diciembre- } \\
2017\end{array}$ & 103 & 33 \\
\hline $\begin{array}{l}\text { 22- diciembre- } \\
2017\end{array}$ & 141 & 40 \\
\hline $\begin{array}{l}\text { 23- diciembre- } \\
2017\end{array}$ & 251 & 99 \\
\hline $\begin{array}{l}\text { 24- diciembre- } \\
2017\end{array}$ & 265 & 84 \\
\hline $\begin{array}{l}\text { 25- diciembre- } \\
2017\end{array}$ & 17 & 3 \\
\hline $\begin{array}{l}\text { 26- diciembre- } \\
2017\end{array}$ & 34 & 6 \\
\hline $\begin{array}{l}\text { 27- diciembre- } \\
2017\end{array}$ & 37 & 8 \\
\hline $\begin{array}{l}\text { 28- diciembre- } \\
2017\end{array}$ & 83 & 39 \\
\hline $\begin{array}{l}\text { 29- diciembre- } \\
2017\end{array}$ & 63 & 19 \\
\hline $\begin{array}{l}\text { 30- diciembre- } \\
2017\end{array}$ & 166 & 56 \\
\hline $\begin{array}{l}\text { 31- diciembre- } \\
2017\end{array}$ & 179 & 61 \\
\hline
\end{tabular}

Fuente: Autores.

Los resultados muestran un nivel de correlación positiva como se puede observar en la figura 9 mostrado a continuación, con un porcentaje cercano al $25 \%$ en el incremento de la cantidad de ventas por recomendaciones efectivas; también existe una mejora significativa en la atención al cliente, la cual, aunque no es medible de forma absoluta es 
importante indicar que el cliente recibe opciones cuya aceptación 0 rechazo permitirá mejorar los modelos desarrollados y en el camino brindar siempre opciones al cliente.

Figura 9. Comparación de Ventas efectivas con ventas generales

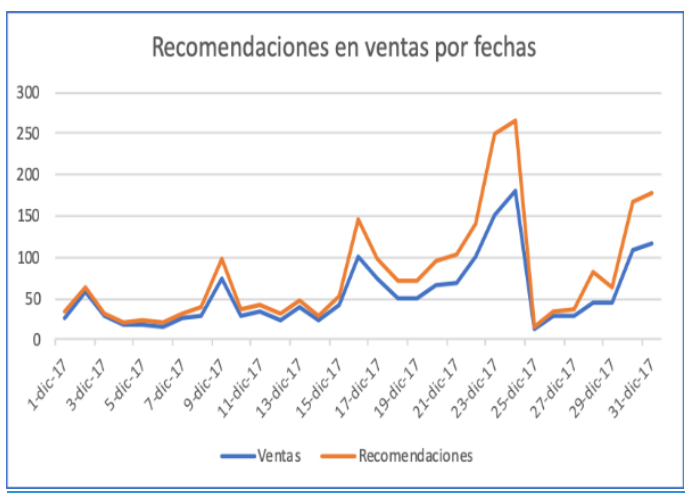

Fuente: Autores.

\section{Conclusiones}

Para la implementación de los algoritmos que normalmente se incluyen en un sistema de recomendación es necesario trabajar con diversidad de formatos y atributos. En el caso de las empresas de tipo retail, se elaboró una estructura jerárquica válida para poder manejar líneas de negocios varías $\sin$ tener que reestructurar la herramienta.

Los algoritmos publicados como un servicio web contribuyen a un nicho de mercado estratégicamente no atendido por las empresas desarrolladores de software. En cuanto a soluciones con aprendizaje automático; son un soporte a las decisiones que, con una arquitectura orientada a servicios, pueden accederse desde cualquier dispositivo con acceso a internet y soporte a servicios web, y entregar múltiples beneficios al sector comercial e investigativo.

La implementación de los algoritmos utilizando microservicios mejora la disponibilidad del servicio como un todo, aislando la funcionabilidad y abriendo las posibilidades a estudios futuros de rendimiento, aplicación y usabilidad de los métodos y algoritmos implementados.

\section{Bibliografía.}

25010, I. (2017). ISO25000. (ISO25000) Recuperado el 01 de 09 de 2017, de http://iso25000.com/index.ph $\mathrm{p} /$ normas-iso-25000/iso25010

Benito Hernández, S. (2009). Las redes de cooperación de microempresas en España y la utilización de las TIC's. CIRIEC-España, Revista de Economía Pública, Social y Cooperativa, 59-84. 
CHANG TAN, Q. L. (2014). ObjectOriented Travel Package Recommendation. $\quad A C M$ Transactions on

Chris Cornelis, J. L. (2007). Oneand-only item recommendation with fuzzy logic techniques. Information Sciences, 906-4921.

Comercio, E. (8 de junio de 2017). Nuevo Tarqui, en la ciudad de Manta, cambió el rostro al comercio. Obtenido de El Comercio:

http://www.elcomercio.com/ac tualidad/tarqui-manta-

comercio-terremoto-

vendedores.html

Dianshuang Wu, G. Z. (2013). A Fuzzy Tree Similarity Measure and Its Application in TelecomProduct

Recommendation.

IEEE International Conference, 3483-3488.

Dryfty. (01 de 09 de 2017). Ionic Framework. (Ionic Enterprise) Obtenido de https://ionicframework.com/

Edgar JuliánGálvez Albarracín, S. C. (2014). Influencia de las tecnologías de la información y comunicación en el rendimiento de las micro, pequeñas y medianas empresas colombianas. Estudios Gerenciales, 355364.

Ignacio Apella, O. A. (2008). Aportes a una nueva visión de la informalidad laboral en la Argentina. Buenos Aires: Banco Mundial; Ministerio de Trabajo, Empleo y Seguridad Social. Intelligent Systems and Technology, 43.

Jie Bao, Y. Z. (2012). Location-based and Preference-Aware Recommendation

UsingSparse Geo-Social Networking Data. Proceedings of the 20th international conference on advances in geographic information systems (págs. 199-208). Redondo Beach, CA, USA: ACM.

López, H. R. (2017). Estudio del Comportamiento en Compras de Clientes de Manabí en una Empresa de Retail un año después del terremoto. INNOVA Research Journal, 126-134.

Mireya Flores Jaén, L. R. (2016). Características de las Pymes familiares en la ciudad de Quevedo. Ecuador. 494-508. 
Monica Orozco, G. Q. (19 de 07 de 2015). El $82 \%$ de pymes de Ecuador accede a Internet, pero su uso se limitaa enviar correos y tareas administrativas. pág. 23.

Navarro, P. (03 de 01 de 2018). las 10 mejores tablets baratas 2018. Obtenido de Comprar Una

Tablet:

https://comprarunatablet.net/t ablets-baratas/

Philippe Fournier-Viger, A. G.-W. (2014). SPMF: A Java OpenSource Pattern Mining Library. Journal of Machine Learning Research, 35693573.

Philippe Fournier-Viger, J. C.-W. (2016). The SPMF opensource data mining library version 2. ECML PKDD (págs. 36-40). Conference Center, Riva del Garda: Springer International Publishing.

School, X. Y. (2017). An improved Apriori algorithm for mining association rules. AIP Conference Proceedings, 080005(1820), 1-6.

Universo, E. (29 de Julio de 2016). El comercio fue el más afectado tras el sismo de 7,8 en Ecuador. Obtenido de El
Universo:

https://www.eluniverso.com/n oticias/2016/07/29/nota/5714 052/comercio-fue-masafectado-tras-sismo-78

Youcef Djenouri, M. C. (2017). SSFIM: Single Scan for Frequent Itemsets Mining in Transactional Databases. PAKDD (págs. 644-654). Jeju, South Korea: Springer International Publishing. 